\title{
¿VIEJOS O NUEVOS? ORIENTACIONES ACTUALES DE LOS SERVICIOS SOCIALES EN ESPAÑA
}

\author{
M. ${ }^{a}$ ASUNCION MARTINEZ ROMAN \\ M. ${ }^{\text {a }}$ TERESA MIRA-PERCEVAL PASTOR \\ HORTENSIA REDERO BELLIDO \\ Profesoras titulares de SS.SS.de la EUTS de Alicante
}

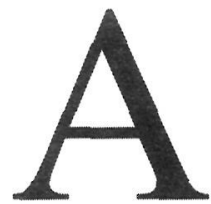

finales de los años setenta, se evidencia en nuestro país una preocupación creciente por imprimir una nueva orientación a las acciones encaminadas a la atención de las necesidades de los sectores más desfavorecidos. Siguiendo las tendencias imperantes en los países de nuestro entorno más próximo con sistemas de Servicios Sociales muy consolidados, se comienzan a oír expresiones en defensa de la «universalidad», la «descentralización», la «participación», la «normalización», la «integración» y otra serie de términos que encierran determinadas concepciones de los derechos humanos y sociales. Desde entonces, estas expresiones han sido defendidas ampliamente como principios de los «nuevos» Servicios Sociales, llegando a incorporarse a las legislaciones autonómicas sobre la materia. No obstante, la evolución seguida por los Servicios Sociales en nuestro país pone de manifiesto que, en la práctica, no siempre se cumplen realmente. En este artículo se propone reflexionar sobre el origen de estos principios y las dificultades que se plantean en su aplicación.

\section{SITUACION DE LOS SERVICIOS SOCIALES \\ EN LA DECADA DE LOS OCHENTA: INDICADIONES PARA SU TRANSFORMACION}

En enero de 1978, tuvo lugar en Madrid, a instancias del entonces denominado Ministerio de Sanidad y Seguridad Social, una consulta a expertos internacionales con el fin de proporcionar al Gobierno español asesoramiento para la reforma de los Servicios Sociales de la Administración Central ${ }^{1}$.

1 «Consulta sobre Política de la Organización y Administración de los Servicios Sociales» convocada por el Ministerio de Sanidad y Seguridad Social, 
Los expertos consultados realizaron una descripción de sus respectivas Administraciones sociales poniendo en evidencia las vinculaciones existentes entre los Servicios Sociales generales y los ámbitos de Salud (tienden a absorberlos) y Seguridad Social (tienden a reducirlos), frente a lo que aconsejaron mantener la necesaria autonomía de los tres ámbitos. Al mismo tiempo subrayaron la importancia de una planificación central, de una financiación plural (descentralización) y de una gestión local de Servicios Sociales integrados (polivalentes), así como la necesidad de favorecer la participación de las fuerzas sociales voluntarias.

Asimismo, los expertos consultados, tras analizar los servicios asistenciales existentes en España en ese momento histórico, hicieron un diagnóstico de la situación que, en síntesis, puso de manifiesto lo siguiente:

1. Que había políticas diversas y paralelas para las diversas necesidades y sectores de población.

2. La existencia de diversidad de organismos especializados, sin conexión entre los distintos departamentos, sin ningún sentido de globalización (los problemas sanitarios parece que no tengan nada que ver con la educación, o ésta con el trabajo, o con el hábitat, etc.).

3. El predominio de la función asistencial y reparadora.

4. Que tradicionalmente la iniciativa privada (frecuentemente la Iglesia Católica) ha ido supliendo al Estado, otras veces se han duplicado servicios, por descoordinación.

5. Los servicios estaban fuertemente centralizados, a pesar del inicio de un lento proceso de descentralización.

6. Algunos sectores de población se encontraban superprotegidos y otros no obtenían la protección necesaria por duplicidad de servicios, habiendo mal uso de recursos y prestaciones desiguales para diversos grupos de población.

7. Mínima participación de la población en los servicios.

El conjunto de todas estas observaciones nos proporcionan una nítida «radiografía» de la situación de los Servicios Sociales en Espa-

que tuvo lugar en Madrid (16-20 enero 1978), en colaboración con el Programa Europeo de Desarrollo Social de las Naciones Unidas. Participaron expertos de Gran Bretaña, Yugoslavia, República Federal Alemana, Noruega, Portugal e Italia además de representantes de organizaciones internacionales (UNICEF, OIT, OMS y AISS). 
ña en ese momento, así como el evidente deseo de reorientarlos para adecuarlos a las nuevas necesidades sociales y al contexto político de la denominada «transición».

Entre otras conclusiones, se indicó que el problema no era tanto la cantidad de recursos como la racionalización de su empleo; que el objetivo primordial debería ser las necesidades y esperanzas de los beneficiarios; y que la centralización y sectorización administrativa hace que algunos sectores de problemas o grupos de población estén desprotegidos y otros sobreprotegidos.

Acorde con el diagnóstico realizado y las sugerencias planteadas, en su informe destacaron la necesidad de reformas urgentes tanto legislativas como institucionales, administrativas y de financiación; reformas estructurales básicas en la administración local; revisión de las relaciones entre las instituciones públicas y no gubernamentales «con el fin de evitar duplicidad de esfuerzos, derroche de recursos y prestaciones desiguales para diversos grupos de población». También se destacó la importancia de «la participación popular en la planificación, organización y transmisión de servicios», entendiendo que ésta debe ser un objetivo esencial de las políticas y programas del Estado ${ }^{2}$.

En la etapa de la transición política, la Administración central y especialmente algunos Ayuntamientos, inician un lento pero paulatino proceso de transformación de los Servicios Sociales, tanto desde el punto de vista organizativo como de orientación de sus actuaciones, tratando de imprimirles el carácter de responsabilidad pública, universales, descentralizados (gestión en el nivel local), normalizadores, y con la participación de los ciudadanos.

En esa misma época (final de los años setenta y comienzo de los ochenta), tiene lugar la convocatoria de numerosas Jornadas, Seminarios, Encuentros, etc., para debatir esta nueva orientación de los Servicios Sociales, apareciendo asimismo diversas publicaciones sobre la materia. En todos estos ámbitos empiezan a analizarse tímidamente por parte de los profesionales de la acción social y políticos, tanto los antecedentes inmediatos como el futuro de dichos servicios ${ }^{3}$.

2 Síntesis del documento United Nations (1979): Policy Consultation on the Organization and Administration of social services in Spain, New York, United Nations, Division of Social Affairs.

3 Algunos ejemplos de las publicaciones de esta época: Casado, D., Cambio de las instituciones de bienestar social en España, Marova, Madrid, 1977; Malla, P. y Arenas, J., Principis inspiradors d'una política de Serveis Socials, Generalitat de Catalunya-Dep. de Sanitat y Assitencia social, Barcelona, 1979; CEBS, Descentralización de los Servicios Sociales, Marsiega, Madrid, 1979; PSOE, Cambio Social y Servicios Sociales, PSOE, Madrid, 1981; Rubiol, G., Los Servicios Sociales: Organización y funcionamiento en Gran Bretaña, Yugoslavia, Israel y Países Bajos, INSERSO, Madrid, 1982; CEBS, Los Servicios 
Sin embargo, la lentitud de este proceso iniciado la podemos constatar. En 1983, Artells hace un diagnóstico de la situación de los Servicios Sociales que se aproxima mucho en sus conclusiones a la realizada por los expertos internacionales:

«1. La concurrencia de legislación y filosofías distintas y contradictorias que han determinado una organización paradójica: a pesar del carácter marginal y de las reducidas dimensiones financieras y estructurales del ámbito de los Servicios Sociales, llama la atención la pluralidad de competencias e instituciones con autoridad presupuestaria y con políticas y acciones escasamente coordinadas.

2. Una estructura de recursos-servicios y equipamientos-caracterizada, en general, por la escasa presencia del sector público y el notable peso de las instituciones de dependencia religiosa o de iniciativa privada no lucrativa, que han aparecido y se han consolidado de espaldas a cualquier intento de ordenación y planificación de cada área de actividad. Dicha falta de presencia activa y de iniciativa del sector público se ha traducido en la orientación predominantemente institucionalizadora de la mayoría de los servicios y centros existentes; o, al menos, de los que absorben la mayor parte de los recursos financieros y humanos disponibles.

3. Un sesgo netamente benéfico y de simple contención de las formas más extremas de necesidad social, basado en las prestaciones económicas de ayuda individual periódica o de emergencia. Este aspecto, perfectamente documentado en lo que se refiere a su peso económico predominante y a la hipoteca que supone para el desarrollo de los Servicios Sociales de contenido técnico, aparece a la vez como vestigio absolutamente presente de la concepción subalterna y paternalista del sistema actual y como una de las principales razones de su burocratización injustificada.

4. La práctica inexistencia de una base informativa sistemática y de renovación regular sobre los aspectos más decisivos de la oferta, utilización, acceso, naturaleza, distribución territorial y contenido de los servicios y equipamientos existentes. Esta precariedad informativa se extiende también al déficit absoluto que existe en relación a los parámetros y estudios básicos para conocer las dimensiones de las distintas necesidades para la población en conjunto y para determinados usuarios en particular.

Asistenciales y Sociales en el Estado de las Autonomías, Marsiega, Madrid, 1983; Generalitat de Catalunya, Mapa de Serveis Socials (5 tomos), Dep. de Sanitat i Seguretat Social, Barcelona, 1984; Colección Serveis Socials, Area de Servicios Sociales del Ayuntamiento de Barcelona; CEBS, Los Servicios Asistenciales y Sociales de Atención Primaria, Marsiega, Madrid, 1984. De esta época hay que destacar numerosos artículos de las revistas especializadas Boletín de Estudios y Documentación de Servicios Sociales. (INSERSO-Ministerio de Trabajo y Seguridad Social) y Cuadernos de Acción Social (Dirección General de Acción Social, Ministerio de Trabajo y Seguridad Social). 
5. La falta de instancias regulares de debate profesional y técnico sobre las necesidades, instrumentos y formación profesional que reclama la adecuación de los recursos existentes a la cobertura de déficit y a la identificación de nuevas necesidades emergentes» ${ }^{4}$.

En 1984 fue convocada una nueva Consulta con el fin de que los expertos internacionales asesoraran al Gobierno español en el Proyecto de Ley de Servicios Sociales que, dentro del ámbito de la Seguridad Social, tenía como objeto «establecer una regulación unitaria e integrada de los Servicios Sociales para todos los ciudadano» ${ }^{5}$.

Los expertos en su informe recomendaron la inclusión, en el borrador de la Ley, de un artículo referente a «Principios Generales», en el que debían considerarse una serie de principios básicos de actuación, relacionados tanto con las responsabilidades de la Administración, como con los posibles beneficiarios. El texto de la recomendación apuntaba los principios que a continuación se mencionan:

a) Relacionados con las responsabilidades de la Administración:

- Planificación.

- Coordinación.

- Descentralización.

- Prevención.

- Normalización.

b) Relacionados con los posibles beneficiarios:

- Participación.

- Desarrollo autocentrado.

- Igualdad de oportunidades.

- Solidaridad.

- Personalización.

La recomendación se hizo con el siguiente texto:

1. Planificación. Los poderes públicos planificarán la prestación de los Servicios Sociales estableciendo los mínimos de prestaciones asistenciales para todo el territorio nacional.

4 Artells, J., (1983) «Notas sobre la crisis económica y el futuro de los Servicios Sociales», Revista de Treball Social, núm. 92, págs. 91-96.

5 Del 5 al 9 de marzo de 1984 tuvo lugar en Madrid la «Consulta Internacional sobre reforma de los Servicios Sociales en España», a petición de la Dirección General de Acción Social, del Ministerio de Trabajo y Seguridad Social y organizada en el marco del programa global de desarrollo social de Naciones Unidas. Una amplia información sobre ella puede encontrarse en: «Consulta Internacional sobre los Servicios Sociales en Españas, Boletín de Estudios y Documentación de Servicios Sociales, núm. 17, 1984, págs. 31-38. 
2. Coordinación. Los poderes públicos coordinarán sus actuaciones y las de la iniciativa social y privada, así como la de diversos organismos estatales entre sí sin perjuicio de las disposiciones adoptadas por las Comunidades Autónomas, a fin de darles un tratamiento unificado.

3. Descentralización. Las competencias sobre Servicios Sociales serán desplazadas al máximo posible hacia los órganos e instituciones de ámbito territorial más limitado.

4. Prevención. Los Servicios Sociales tenderán no sólo a remediar las situaciones existentes de marginación, sino también y, sobre todo, a prevenir las causas de la marginación.

5. Normalización. Los Servicios Sociales facilitarán a los ciudadanos el acceso a las instituciones de carácter general, salvo cuando por sus características personales requiera una atención especial.

6. Participación. Los poderes públicos fomentarán la participación democrática de los ciudadanos en la planificación y control de los servicios públicos a través de los mecanismos que establece la Ley.

7. Desarrollo autocentrado. Los poderes públicos estimularán la constitución de organizaciones de autoayuda y les prestarán asistencia técnica y colaboración financiera.

8. Solidaridad. Los poderes públicos estimularán la solidaridad en las relaciones entre las personas y los grupos sociales con vistas a superar las condiciones que crean marginación.

9. Igualdad de oportunidades. Los poderes públicos, basados en el concepto de igualdad entre todos los ciudadanos, promoverán actuaciones y legislación destinadas a garantizar una efectiva igualdad de oportunidades para las mujeres, los jóvenes, los impedidos y otros sectores necesitados.

10. Personalización. Los poderes públicos promoverán el bienestar de los ciudadanos tanto en la dimensión individual como comunitaria.

Si bien la citada Ley no llegó a ser una realidad, este documento reitera las recomendaciones anteriormente expuestas, basadas en las líneas de acción imperantes en materia de Servicios Sociales en el panorama internacional, especialmente, en los países nórdicos y Gran Bretaña. No obstante es de destacar que estas orientaciones sí han sido recogidas de forma unánime por las respectivas leyes que regulan los Servicios Sociales en las distintas Comunidades Autónomas que, con mayor o menor énfasis en algunos de ellos, incluyen en sus textos el reconocimiento de estos principios. 


\section{CONSIDERACIONES ACERCA DE LA APLICACION PRACTICA DE LOS PRINCIPIOS DE LOS SERVICIOS SO- CIALES: OBSERVANDO A OTROS PAISES}

Como es obvio, la asunción de estos principios implica, necesariamente, poner en práctica modos de respuesta ante las necesidades sociales muy diferentes de los tradicionales, exige importantes cambios de mentalidad y priorizar determinadas alternativas frente a otras. La experiencia de otros países nos puede ayudar a reflexionar sobre ello.

\subsection{Responsabilidad Pública}

Existe un amplio consenso en torno a la idea de que la Administración Pública debe asumir plenamente su responsabilidad frente a los problemas objeto de la acción de los Servicios Sociales, facilitando y promoviendo los recursos financieros, técnicos o institucionales necesarios para garantizar unas prestaciones mínimas para todo el territorio. Asimismo se defiende que el Estado debe salvaguardar el derecho a la igualdad de todos los españoles, superando el papel de subsidiariedad en la atención a las necesidades que tradicionalmente había desempeñado en España.

No obstante, «responsabilidad pública» no quiere decir, necesariamente, que los servicios deban ser de titularidad y gestión pública. Así podemos constatar como en Inglaterra, la nueva legislación aprobada por el Parlamento en 1990 y en vigor desde 1991, propone, entre otras medidas, que los municipios acuerden con compañías privadas, agencias y asociaciones la prestación de los servicios de carácter residencial ${ }^{6}$. De este modo, los municipios, estando obligados por ley a atender las necesidades sociales de sus ciudadanos, juegan un rol de mediadores más que de suministradores de servicios e introducen cada vez más mecanismos selectivos de modo que todo aquel que disponga de medios económicos contribuya económicamente. Este hecho provoca que las organizaciones no lucrativas y voluntarias se vean obligadas a ofrecer mejores y más flexibles servicios para competir en el mercado cuando, paradójicamente, han visto

6 Dos son los documentos básicos para conocer las nuevas orientaciones en Inglaterra, el «libro blanco» (basado en el Informe Griffith), que fue presentado al Parlamento en noviembre de 1989: Caring for people. Community Care in the next decade and beyond. HMSO, Londres, 1990. Y la guía de aplicación de la «National Health Service and Community Care Act 1990»: Caring for people. Community Care in the next decade and beyond, Police Guidance, HMSO, Londres, 1990. 
drásticamente recortada la financiación que precisan. Ciertamente las Asociaciones podrían proporcionar buenos servicios basados en los principios orientadores de los Servicios Sociales modernos si el Estado les apoyara económicamente, lo que debe ser tenido en cuenta por la Administración.

En esta misma línea, la defensa que actualmente se hace de favorecer al máximo la permanencia del individuo en su propio entorno, evitando al máximo desvincularlo de lo que es su hábitat natural y apoyándolo para que consiga vivir de forma autónoma, implica una exigencia importante para la Administración, en el sentido de que ésta debe proporcionar los recursos necesarios a nivel comunitario y facilitar a las familias los apoyos necesarios para la atención de sus miembros dependientes. Sin embargo, los pasos que se han dado en este sentido en nuestro país evidencian que, en la práctica, es muy frecuente que se «descarguen» todas las obligaciones en las familias de la persona que precisa cuidados, sin tener en cuenta los importantes cambios acaecidos en dicha institución y las necesidades que genera en la familia el hecho de tener un miembro que necesita cuidados continuados ${ }^{7}$.

\subsection{Universalidad}

Un debate clásico en política social es el que enfrenta a los partidarios de unos Servicios Sociales dirigidos a todos, como un derecho ciudadano, con los partidarios de la creación de servicios sólo para aquellas personas que, por diferentes razones, no pueden satisfacer por sí mismas sus necesidades. La elección de la «universalidad» implica que se han de ofrecer no sólo servicios para «necesitados», sino para todos los ciudadanos, más allá del clásico planteamiento de servicios selectivos para determinados sectores $y / o$ categorías de población.

Con la oferta de servicios universales, se quiere subrayar la igualdad de los ciudadanos, tratando de evitar la estigmatización que supone el reducir la oferta a personas con deficiencias de índole física, psicológica o social. Pero esto no ha de ser óbice para ofrecer servicios diferenciados ante especiales situaciones que así lo demanden ${ }^{8}$.

${ }^{7}$ Este problema se analiza en Martínez Román, M. ${ }^{a}$ A.; Mira-Perceval Pastor, M. ${ }^{a}$ T. y Redero Bellido, H., «Función específica de la familia en la atención social comunitaria: su valor como recurso para la sociedad», Alternativas. Cuadernos de Trabajo Social, núm. 1, 1992, págs. 33-53.

8 Williams, F., «Somewhere over the rainbow: Universality and diversity in social policy», en Manning, N. y Page, R. (ed.), Social Policy Review 19911992, Social Policy Association, Canterbury, 1992, pág. 16. 
Existen grupos de población con necesidades específicas que precisan respuestas más ajustadas a su especial situación, ya que los servicios «universales» no responden con la cualificación que sus necesidades exige. Esto ha provocado el surgimiento de diversos movimientos asociativos que reivindican una mayor atención de la sociedad hacia sus problemas y el reconocimiento de la diversidad y la diferencia. La selectividad en este sentido puede significar una discriminación positiva a favor de estas personas proporcionándoles unos servicios «puente» que les faciliten el acceso posterior a los servicios universales ${ }^{9}$.

A partir de los ochenta, como consecuencia de la crisis económica, se han producido importantes recortes de los presupuestos públicos en materia de gastos sociales en países con sistemas de bienestar muy consolidados. Los partidarios de ofrecer sólo servicios de modo «selectivo» tratan de limitar la oferta de servicios a aquellas personas «que lo necesiten», midiendo la «necesidad» en términos meramente económicos, es decir, si disponen o no de medios económicos para pagar por ellos. Estos planteamientos han originado un descenso en la cantidad y calidad de los Servicios Sociales públicos y privados no lucrativos de estos países, así como un auge de los servicios privados lucrativos, con el consiguiente aumento de la desigualdad social ${ }^{10}$.

El debate entre «universalidad»/«selectividad» de los Servicios Sociales continúa abierto, con defensores de una u otra opción, si bien, como señalan diversos autores, la «universalidad» por sí sola no significa igualdad (en términos de estatus), ni el hecho de ofrecer servicios «selectivos» para determinados grupos y en determinadas condiciones, significa necesariamente estigmatización ${ }^{11}$.

\subsection{Descentralización}

Hasta los años setenta los Servicios Sociales tendían a estar fuertemente centralizados y la experiencia de muchos países avaló la conveniencia de aproximar los servicios al ciudadano con el fin de 135.

9 Titmuss, R., Commitment to Welfare, Allen \& Unwin, Londres, 1968, pág.

${ }^{10}$ McCarthy, M. (ed.), The New Politics of Welfare: An Agenda for the 1990s?, Macmillan, Londres, 1989, pág. 124. Entre los muchos autores que comparten esta tesis ver: Raison, T., Tories and the Welfare State, Macmillan, Londres, 1990 y Johnson, N., Reconstructing the Welfare State, Harvester/Wheatsheaf, Londres, 1990.

${ }^{11}$ Jones, K.; Brown, J.; Bradshaw, J., Issues in Social Policy, 2. ${ }^{a}$ ed., Routledge \& Kegan Paul, Londres, 1983, págs. 46-61. 
prestar una mejor atención a las necesidades allí donde éstas se producen. El término hace referencia tanto a la descentralización administrativa de las competencias en materia de Servicios Sociales (gestión en el nivel local), como a la descentralización en la organización de los propios departamentos de Servicios Sociales, potenciando el trabajo de comunidad.

La descentralización administrativa supone la transferencia de las competencias relacionadas con la planificación, gestión y financiación de los servicios desde la Administración Central hacia la Administración Regional y Local con la finalidad de conseguir una mayor eficacia. En materia de Servicios Sociales se arguye que la descentralización administrativa es un modelo organizativo que trata de superar las disfunciones del modo centralizado que implica un tratamiento uniforme de las necesidades, lejos de los interesados y sin contemplar las diferencias culturales, sociales, geográficas, etc.

Con los Servicios Sociales descentralizados en pequeñas unidades locales, se plantea el cambio de la atención individual, de casos, al énfasis en el trabajo con grupos y comunitario y a la fusión entre la acción de los servicios públicos y las organizaciones voluntarias en la comunidad. Son conceptos clave: nivel local, participación, ayuda informal y voluntariado ${ }^{12}$.

Esta mayor cercanía de los servicios a los individuos y grupos que los demandan, se justifica aduciendo que permite un mejor conocimiento de los problemas, la posibilidad de proporcionar respuestas más adecuadas a las necesidades sociales locales y una mayor participación directa de la comunidad en la toma de decisiones. Para que todo esto sea posible hay que desplazar las competencias hasta el ámbito territorial más limitado. El único límite a la descentralización es la necesidad de una igualdad básica de servicios y prestaciones a las que tienen derecho todos los ciudadanos de un Estado, independientemente de su comunidad de residencia.

Las críticas e interrogantes a la práctica de la descentralización han sido numerosas. Entre las más recientes se encuentran aquéllas que ponen evidencia que si bien la descentralización de los servicios es necearia para facilitar la participación de la población, sin embargo, la existencia de unos servicios descentralizados no la garantiza, ni asegura la igualdad real de oportunidades. En otras palabras, la descentralización es requisito necesario pero no suficiente para una auténtica participación. En definitiva se plantea la distinción entre la descentralización en el sentido de localizar los servi-

12 Auriol, D., «L'autonomisation des politiques sociales d'insertion, 19701990. L'emergence d'une nouvelle catégorie d'action», Revue Française des Affaires Sociales, 47 ann., núm. 1, 1993, págs. 41-63. 
cios en la propia comunidad, y la descentralización en el sentido de democratización de los servicios ${ }^{13}$.

\subsection{Normalización}

La integración social debe facilitarse a todos los ciudadanos, haciendo posible el acceso a los servicios de carácter general, salvo que se requiera una atención especial. De acuerdo con este principio, la atención que se preste a los individuos que presentan dificultades para su desarrollo personal y social, debe llevarse a cabo a través de los servicios establecidos como generales para la satisfacción de las necesidades sociales. La historia de la acción social nos proporciona numerosos ejemplos de cómo la utilización de servicios o centros considerados «especiales» o de exclusiva atención a determinados problemas o grupos de población, crean modos de vida a espaldas de la sociedad, con lo que se consigue reforzar el sentimiento de «diferencia» alejando la posibilidad de una integración/reinserción social.

El aislamiento de las personas de su medio ambiente habitual dificulta e impide a medio y largo plazo su integración en la comunidad, con todas las consecuencias negativas de índole físico, psíquico y social que ello tiene. Salvo en aquellos casos en que su total normalización sea prácticamente imposible, se debería potenciar el acceso y uso por estos sectores de los servicios y recursos utilizados por el resto de la población.

Los antecedentes de este principio se encuentran en los países escandinavos, en donde se llevaron a cabo experiencias de «normalización» para favorecer la integración de los deficientes mentales ${ }^{14}$, experiencias que más tarde se han generalizado a otras personas en situación de desventaja social. Nirje señala que no se trata de normalizar a la persona, sino «normalizar las condiciones de vida», por su

13 Los autores, de la corriente «radical» del Trabajo Social, plantean la descentralización como condición indispensable para la democratización. Esta se concreta en una participación real de la población, es decir, en la toma de decisiones, lo que implica una «redistribución del poder». Ver, por ejemplo, Croft, S. y Beresford, P., «Decentralization and the Personal Social Services», en Langan, M. y Lee, P., Radical Social Work Today, Unwin Hyman Ltd., Londres, 1989, pág. 97-119.

14 Neils, E. Bank Mikkelsen, ya en 1959, consigue en Dinamarca que se incluya en la legislación el concepto de «normalización» aplicado al campo de la deficiencia mental. En 1959 la define como la «posibilidad de que el deficiente mental desarrolle un tipo de vida tan normal como sea posible». Pero es en 1969 cuando se formula sistemáticamente por Bengt Nirje: «hacer accesibles al retrasado mental patrones y condiciones de vida diaria tan semejantes como sean posibles a las normas y patrones en la sociedad en general». 
relación causal con los retrasos impuestos o adquiridos. Wolfensberger, en los años setenta, la define com «el uso de medios lo más normativos posibles desde el punto de vista cultural para establecer y/o mantener comportamientos y características personales que sean de hecho lo más normativas posibles» ${ }^{15}$. El mismo autor, posteriormente, señala las trabas que la sociedad misma pone al desarrollo de un niño con deficiencias, destacando el hecho de que los límites sociales son mayores que los personales y continúan a lo largo de la vida del sujeto, por lo que propone sustituir el término «normalización» por el de «valoración del rol social», que implica «la creación, apoyo y defensa de roles sociales valiosos para las personas de alto riesgo de devaluación social» ${ }^{16}$; esto será una realidad si se refuerzan tanto su imagen social como sus capacidades personales. Más recientemente Nirje, autor clave en este tema, analiza las dificultades que se encuentran en la práctica y nos alerta acerca de las concepciones de este principio que se desvían de su esencia ${ }^{17}$.

En definitiva, el valor básico de la normalización es el énfasis en asumir que las personas con alguna deficiencia tienen las mismas potencialidades que cualquier otra no afectada, pero necesitan recibir apoyo y ayuda para que esas potencialidades se desarrollen realmente.

Un importante campo de aplicación de este principio de normalizacion es la educación, área en la que tradicionalmente se optaba por la segregación de las personas con necesidades «especiales» para posteriormente favorecer su integración en el sistema educativo general, lo que implica políticas específicas acompañadas de suficientes recursos para hacerlas efectivas ${ }^{18}$. Otro importante ámbito para la aplicación de este principio es el de las personas con problemas de salud mental, tradicionalmente segregadas y a las que actualmente se intenta «mantener en la comunidad» no siempre con los

15 Wolfensberger, $\mathrm{W} .$, The Principle of normalization in human services, National Institute on Mental Retardation, Toronto, 1972.

${ }^{16}$ Wolfensberger, W., Normalization: The principle of Normalization in Human Services, National Institute for Medical Research, Londres, 1982; «Social role valorization: a proposed new term for the principle of normalization», Mental Retardation, vol. 21, núm. 6, 1983, págs. 234-239, y «A reconceptualization of normalization as social role valorization», The Canadian Journal of Mental Retardation, vol. 34, núm. 2, 1984.

17 Perrin, B. y Nirje, B., «Setting the record straight: a critique of some frequent misconceptions of the normalization principle», Australia and New Zealand Journal of Developmental Disabilities, vol. 1, núm. 1, 1985, págs. 69-74.

${ }_{18} \mathrm{~L}$. Barton recopila los trabajos de una Conferencia Internacional en relación al tema de la integración en educación proporcionando un análisis comparativo de las políticas en vigor. Barton, L. (ed.), Integration: Myth or reality, Falmer, Londres, 1989. 
servicios de apoyo que precisan. Estos servicios pueden ser de diferente índole según vayan dirigidos a los cuidadores (grupos de auto ayuda, centros de voluntarios, hospital de día, formación de cuidadores, servicios para proporcionar apoyo temporal, etc.), o más directamente a las personas dependientes para proporcionarles cuidados o atenciones ${ }^{19}$.

Las implicaciones derivadas de la deficiente aplicación de este principio en la prestación de servicios a aquellas personas que están en situación de desventaja por distintos motivos, respecto a la población considerada «normal», son objeto de análisis y suscitan importantes críticas ${ }^{20}$. La mayoría de estudios concluyen subrayando la falta de apoyo real para estas personas y, en consecuencia, las limitaciones existentes para hacer efectiva su integración social.

\subsection{Participación}

Tal como se ha enunciado al hablar de la descentralización, una de sus justificaciones es la posibilidad de hacer viable la participación de la población en este campo, en el sentido de que la planificación de los Servicios Sociales no puede venir dada únicamente desde fuera de las comunidades a las que van dirigidos. La comunidad debe poder integrarse en el estudio y el análisis de sus necesidades, en las soluciones y en el control de la gestión de los recursos económicos e institucionales.

Ya en 1979 el Comité Directivo de Asuntos Sociales del Consejo de Europa señalaba que, la evolución de la acción social había supuesto un giro radical de la figura del beneficiario, que debía pasar a ser sujeto y no mero objeto por lo que instaba a promover la participación de los usuarios de los Servicios Sociales:

19 Atkinson, D. y Ward, L., «Friends and Neighbours: relationships and opportunities in the community for people with a mental handicap», en Malin, N. (ed.), Reassessing Community Care, Croom Helm, Londres, 1987; Twigg, J. et al., Carers and services: A review of research, HMSO, Londres, 1990, págs. 12-66.

20 Como ejemplos tenemos, la obra de Flynn, R. J. y Nitsch, K. E. (eds.), Normalisation, Social Integration and Community Services, University Park Press, Madison, 1980; más concretamente, aplicado a la formación de trabajadores sociales, el trabajo de Ramón, S., que sugiere los conocimientos y destrezas necesarios para el Trabajador Social en «Skills for normalisation work», Practice, vol. 2, núm. 2, 1988, págs. 139-149; o más recientemente, una evaluación del interesante «Barnados' Croxteth Park Project», de dependencia municipal, para niños deficientes mentales profundos, en Alaszewski, A. y Ong, N. (ed.), Normalisation in Practice, Routledge, Londres, 1990. 
«participación de los usuarios» es la posibilidad de que el usuario influya realmente en la política de los SS.SS. en cualquiera (o en todos), de los estadios del proceso de planificación, decisión, puesta en práctica y evaluación, incluido el control de la utilización que se hace de la contribución de los participantes. Esta participación puede ser directa o indirecta e ir de la consulta a la participación en las decisiones.»

Un Informe de las Naciones Unidas realizado en el mismo año destaca la conveniencia de promover programas basados en la comunidad que permitan iniciativas, formulación de decisiones y actividades de autoayuda por parte de la población. También reconoce el valor de la participación de los ciudadanos y el cambio cualitativo que supone «el paso de beneficiarios pasivos (usuarios/clientes) al papel de asociados activos en el desarrollo de la comunidad y en la autorrealización personal ${ }^{21}$. Las cuestiones esenciales que se plantean son «participación popular, para qué», «participación popular, ejercida por quiénes» y «cómo hacer la participación» 22.

La aplicación de este principio plantea problemas de organización de los Servicios Sociales y, de hecho, hay estudios que evidencian las dificultades que se suscitan en la práctica y cómo, con frecuencia, no es posible el participar en la toma de decisiones a todos los trabajadores, todos los usuarios y todos los miembros de la comunidad local ${ }^{23}$. Es decir, la participación precisa un contexto organizativo que la facilite y un espíritu democrático. Si bien existen evidencias de las ventajas de la participación en los Servicios Sociales en cuanto que al favorecer la autodeterminación y la autonomía de las personas evitan la dependencia, también hay que decir que no es una «varita mágica» y que puede tener unos límites ${ }^{24}$.

El tema de la participación no está cerrado; por el contrario, parece que actualmente existe una preocupación creciente por llegar a fórmulas alternativas que favorezcan su presencia. En esta línea se

21 Naciones Unidas, «Los Servicios Sociales en el desarrollo rural. Problemas relacionados con su diseño y prestación», Boletín de Estudios y Documentación de Servicios Sociales, núms. 18-19, 1984, págs. 64-67.

${ }^{22}$ Martner, G. y Rao, M., «Consulta Internacional sobre los Servicios Sociales en España», Boletín de Estudios y Documentación de Servicios Sociales, núm. 17, 1984, pág. 38.

23 Webb, A. y Wistow, G., Social Work, Social care and Social Planning: The Personal Social Services since Seebohm, Essex, Longman, 1987, Págs. 116-122; Bernstein, S., «What happened to self-determination?», Social Work with Groups, vol. 16, núms. 1-2, 1993, págs. 3-14.

24 A. Richardson analiza los objetivos y límites de la participación en la política social en su libro: Richardson, A., Participation, Routledge \& Kegan Paul, Londres, 1983. 
sitúa el denominado welfare mix como sistema de bienestar mixto que empieza a generalizarse en Europa ${ }^{25}$.

El modelo de welfare mix defiende la necesaria recuperación del «tercer sector» (instituciones solidarias y/o voluntarias: familia extensa, organizaciones informales, corporaciones, cooperativas, asociaciones de ayuda mutua, voluntariado...) en las tareas de acción social para responder a las necesidades sociales de la población. Se recupera el protagonismo de la sociedad civil subrayando su importante papel y no sólo como un remedio a la actual crisis económica ante la imposibilidad de los gobiernos de atender todas las demandas sociales de los ciudadanos. Este planteamiento no cuestiona la importancia del Estado y su responsabilidad en la atención social de los ciudadanos, ya que reprueba a aquellos gobiernos que tratan de compartir su ineludible responsabilidad con la sociedad civil sólo para reducir el gasto social. Así, se ha criticado que la ideología del mercado libre, para «contraer las fronteras del Estado», en algunos países potencia y elogia el papel del sector voluntario de modo engañoso. El riesgo evidente es que la participación del denominado tercer sector se convierta en un instrumento útil para ocultar aspectos más severos de una política social restrictiva ${ }^{26}$.

Es de destacar asimismo, un movimiento creciente de reivindicación de los derechos ciudadanos, entre los que se incluyen los derechos sociales ${ }^{27}$, que coincide con un cambio de terminología para referirse a los tradicionalmente denominados «clientes» de los Servicios Sociales. En efecto, parece que la preocupación por la participación afecta incluso a los términos que utilizamos, así parece que hablar de «clientes» está en desuso porque recuerda épocas pasadas que se pretenden superar, de forma que, en la literatura profesional, se rehúye el término sustituyéndolo por el de «usuarios». No obstante, este último también ha sido objeto de críticas porque, en opinión de sus detractores, refuerza la idea de mero consumidor de servicios y porque, en el fondo, se contempla a la población en términos de usuarios potenciales, como posibles beneficiarios de cuidados 0 atención y no capaces de «autocuidarse», de ser independientes ${ }^{28}$.

25 Evers, A. y Wintersberger, H. (eds.), Shifts in the Welfare Mix. Their on work, social service and welfare policies, European Centre for Social Welfare Policy and Research, Frankfurt, 1990.

${ }^{26}$ Maxwell, S., «El sector voluntario escocés y su relación con los gobiernos central y local», en II Seminario Internacional de Política Social «Gumersindo de Azcárate», Madrid, marzo de 1992.

27 Van Steenbergen, B., «Scenarios for Europe in the 1990s: The Role of Citizenship and Participation», Futures, vol. 22, núm. 9, 1990, págs. 961-969.

28 Sugerencias sobre una organización democrática de los Servicios Sociales, que favorezca la participación real de la población pueden encontrarse en: Beresford, P. y Croft, S., Whose Welfare: Private care or public services?, Lewis Cohen Urban Studies Centre, Londres, 1986. 
La integración social implica la posibilidad de participación en la vida social y esto, en el caso específico del Trabajo Social, significa el reconocimiento de las capacidades intrínsecas de toda persona (incluso las que tienen deficiencias de cualquier tipo) para vivir de forma autónoma y resolver sus problemas, aun cuando precisen ayuda para ello, evitando generar dependencia y la cronificación de situaciones. Cuando no existe este reconocimiento del «rol social valioso» de toda persona, en el sentido comentado al hablar del principio de normalización, la práctica profesional favorece la discriminación y se aumentan los obstáculos para la integración social de determinadas personas en lugar de ayudarles a salvar todos los ya existentes. Esta actitud puede hacerse extensiva a las diferentes profesiones que ejercen su trabajo en los Servicios Sociales.

En Gran Bretaña existe un creciente movimiento entre Trabajadores Sociales que critica el uso que una mayoría de estos profesionales han hecho del poder que la legislación les otorga y proponen un cambio radical en las relaciones trabajador social/cliente-usuario en el sentido de permitir realmente a toda persona que sea sujeto de la acción profesional y no mero objeto. Aducen que se ha abusado del estatus profesional para decidir sobre importantes aspectos de la vida cotidiana de las personas sin permitirles una participación activa y entienden que el Trabajo Social, por el contrario, debe involucrar a las personas en el proceso de resolución de sus problemas con el fin de evitar dependencia. El Proyecto de Atención Comunitaria de personas con problemas de salud mental de los Servicios Sociales Municipales de Chesterfield, es un buen ejemplo de cómo el incremento de la participación de los propios usuarios en los servicios, puede ayudarles a prevenir o superar situaciones de dependencia. En dicho proyecto, basado en las nuevas orientaciones del Trabajo Social, se identifica la posibilidad de participación con el acceso real a los diferentes niveles de poder, considerando al grupo como depositario. Ello implica una organización en la que sólo tienen posibilidad real de participar un pequeño número de privilegiados. Se pretende explícitamente que las personas con problemas de salud mental, constituidas en miembros de un grupo de ayuda mutua, desarrollen las destrezas y habilidades necesarias para su mejor integración familiar, social y, si es posible, laboral. Para ello se entiende que deben participar absolutamente en todo, es decir, no sólo en la gestión y evaluación de los servicios, sino también en la misma planificación. Y esto que puede parecer afecta sólo al funcionamiento interno del grupo, se extiende a las relaciones del grupo con la comunidad, incluyendo las funciones de grupo de presión demandando servicios a las autoridades locales y llevando a cabo tareas de sensibilización social acerca de los problemas del propio colectivo. 


\subsection{Prevención}

Las tendencias de los países que nos sirven de marco de referencia coinciden en primar los programas de prevención sobre los de mera asistencia y rehabilitación, estableciendo los servicios adecuados para ello. Es más rentable, social y económicamente, prevenir los problemas sociales que tener que crear recursos sociales para atender las necesidades cuando éstas ya los han generado. En efecto, determinados problemas sociales se pueden evitar con una acción preventiva eficaz. Por ejemplo, cada día se insiste más en la necesidad de actuar preventivamente en el campo de los menores ${ }^{29}$, tanto para evitarles deprivaciones como para romper el posible círculo en el que podrían quedar atrapados ante situaciones como pobreza o malos tratos, que, llegada la edad adulta, podría llevarles a reproducir, al crear su propia familia, las situaciones en las que se han visto inmersos en sus familias de origen ${ }^{30}$.

Al hablar de prevención en Servicios Sociales hay que distinguir tres modos de intervención: la prevención «primaria», que supone actuar tratando de influir en las estructuras ideológicas, económicas y políticas de la vida social que tienden a aumentar la posibilidad de la pobreza y la deprivación (tiene, lógicamente, implicaciones más allá de los Servicios Sociales); la prevención «secundaria», es decir, la actuación en situaciones de riesgo inmediato en las que todavía no se ha producido un daño pero se observa una combinación de circunstancias que, de no modificarse a través de las intervenciones adecuadas, puede llegar a producirse; y la prevención «terciaria», que hace referencia a aquella que se trata de evitar la recurrencia de un comportamiento de necesidad o sufrimiento socialmente inaceptable ${ }^{31}$.

Tradicionalmente, los Servicios Sociales y el Trabajo Social han centrado sus actuaciones en la prevención terciaria. Los clientes han acudido a los servicios por alguna dificultad social y la función de los servicios ha sido reparar la situción y, en lo posible, prevenir que vuelva a producirse. Una actuación social preventiva, para que sea eficaz, nos lleva de nuevo a la necesidad de potenciar el trabajo de orientación comunitaria $\mathrm{y}$, por consiguiente, a destacar el principio

29 Segal, E. y Gustavsson, N., «The High Cost of Neglecting Children: The Need for a Preventive Policy Agenda», Child \& Adolescent Social Work Journal, vol. 7, núm. 6, 1990, págs. 475-485.

${ }_{30}$ Kahn, A. y Kamerman, S., «Do the Public Social Services have a future?», Families in Society: The Journal of Contemporary Human Services, vol. 71, núm. 3, 1990, págs. 165-171; Bush, M., Families in distress. Public, private and civic responses, Berkeley, University of California Press, Los Angeles, CA, 1988, cap. 7.

31 Leonard, P., «The challenge of primary prevention», Social Work Today, vol.2, núm. 5, 1971. 
de la descentralización. Implica un nuevo enfoque de las actuaciones superador de la microintervención centrada en el individuo y su familia, para centrarse más en la comunidad, con un planteamiento más sistémico ${ }^{32}$ y sin olvidar los posibles dilemas éticos que se pueden plantear. Por ejemplo, en el caso de menores en peligro de abandono o malos tratos, los Servicios Sociales tienen posibilidad de actuar antes de que se produzcan daños graves, pero esto puede suponer la separación del niño de su familia natural y ello tiene también consecuencias negativas para el menor, por lo que la elección no siempre es fácil ${ }^{33}$.

El trabajo preventivo no puede plantearse de una manera directiva desde los Servicios Sociales, por el contrario, hay que partir de unas relaciones horizontales entre éstos, otras instituciones sociales y grupos profesionales, y la misma comunidad en la que éstos trabajan.

\subsection{Coordinación}

La coordinación es necesaria para evitar la dispersión de recursos sociales y lograr una mayor efectividad de los existentes, racionalizando la actividad en este área ${ }^{34}$. Se refiere, por una parte, a la coordinación de la propia Administración pública tanto en un mismo nivel como entre sus diferentes niveles (central, autonómico y local) y por otra, a la coordinación de la Administración con la iniciativa privada (social y lucrativa) en la línea que ya hemos dibujado del welfare mix.

El primero de los casos plantea, por ejemplo, la creación de comisiones interministeriales o interconsejerías (o concejalías) para elaborar legislación o planes de acción específicos que supongan la actuación de varios departamentos ministeriales y organismos con competencias en un mismo tema (por ejemplo, el Plan de Igualdad de

${ }^{32}$ Sainsbury, E., The Personal Social Services, Pitman, Londres, 1987, pág. 145.

${ }^{33}$ En Gran Bretaña, los Departamentos de Servicios Sociales municipales tienen establecidas unas listas de menores en riesgo a los que se les presta especial atención. El problema es que sus familias quedan catalogadas como «familias problema» lo que es criticado por algunos Trabajadores Sociales que consideran esta práctica lesiva para los ciudadanos.

${ }^{34}$ Neugeboren, B., «Introduction: Coordinating human service delivery», Administration in Social Work, vol. 14, núm. 4, 1990, págs. 1-10.

${ }^{35}$ McAuley, W. J. y Safewrigth, M. P., «Formal and informal interaction between area agencies on aging and departments of social services: implications for practice», Journal of Gerontological Social Work, vol. 18, núms. 1/2, 1991, págs. 97-115; Beatrice, D., «Inter-agency coordination: A practitioner's guide to a strategy for effective social policy», Administration in Social Work, vol. 14, núm. 4, 1990, págs. 45-60. 
Oportunidades de la Mujer). Con esto se evita que los mismos problemas sean abordados de forma distinta por servicios diversos ${ }^{35}$. El segundo se refiere a la conveniencia de unificar criterios relativos a los objetivos de la acción social por parte de los tres trípodes del sistema de welfare moderno con el fin de racionalizar los medios empleados en aras a conseguir mejores resultados y velar por una mayor calidad de los servicios: cooperación entre las tres partes.

Otro aspecto de la coordinación es la integración de los propios Servicios Sociales, con el fin de poder dar una respuesta global a las necesidades. La coordinación se relaciona con diferentes modelos de organización de los servicios, lo que nos lleva a viejos debates, como es el de primar la polivalencia o la especialización. La especialización implica «una forma de organización de los Departamentos de Servicios Sociales de forma que el trabajo se divide en categorías y los Trabajadores Sociales que están en secciones de la organización asumen el trabajo sólo o principalmente relativo a las categorías de cualificación que su sección acepta» ${ }^{36}$.

En Gran Bretaña, los departamentos de Servicios Sociales estaban especializados, en cuanto a la división del trabajo, entre servicio de base (barrio), a domicilio, atención de día y residencial, y dentro de estas categorías, por la división de casos entre grupos de clientes. Esta modalidad organizativa supone fragmentación, en el sentido de que estas subdivisiones de especialización estaban frecuentemente separadas por límites organizacionales. Este esquema de organización tiene sus raíces en el tradicinal modelo burocrático, según el cual centralización y jerarquía son esenciales al sistema, y especialización y gran escala constituyen características de la eficiencia del sistema ${ }^{37}$.

En los últimos años ha habido críticas respecto a la «eficacia» y a las «razones políticas» de esta modalidad organizativa, lo que ha provocado que, desde los ochenta, se introdujeran importantes modificaciones en la organización del trabajo de los equipos de Servicios Sociales, en muchos casos, dentro de las estructuras tradicionales. El cambio consistió en dedicar un equipo para un territorio determinado, esto es, el área subdividida en subáreas, de forma que se haga posible un trabajo de orientación más comunitaria buscando establecer/fortalecer las redes de atención y las organizaciones voluntarias, con los equipos de salud primaria, escuelas, y policía.

36 Payne, M., Power, Authority and Responsability in Social Services, MacMillan Press, Londres, 1979, pág. 169.

37 Hadley, R.; Dale, P. y Sills, P., Descentralising Social Services. A model for change, Bedfore Square, Londres, 1984, págs. 1-19. 


\section{REFLEXIONES EN TORNO A LA SITUACION ACTUAL DE LOS SERVICIOS SOCIALES EN ESPAÑA}

La situación de los Servicios Sociales en nuestro país analizada a la luz de los principios orientadores expuestos en el primer apartado de estas páginas, nos sugiere las siguientes consideraciones:

- Tras la aprobación de la Constitución y, fundamentalmente desde que asume el gobierno el PSOE, el Estado ha ido progresivamente asumiendo la responsabilidad que le corresponde en la atención de las necesidades sociales, tratando de convencer a la población de que los servicios públicos no debían seguir identificándose con el paternalismo y la beneficencia. En algunas Comunidades Autónomas esto no ha impedido una colaboración y coordinación con la iniciativa privada no lucrativa, pero en otros casos parece que se ha querido «borrar» el pasado, olvidando las importantes acciones que había desarrollado la iniciativa social en este campo, relegándola a un papel meramente subsidiario. Por el contrario, en los últimos años se viene observando un giro significativo ya que, por una parte, el Estado parece querer destacar de nuevo la actuación de la sociedad civil en el campo de la acción social pero, por otra, no le proporciona los medios financieros necesarios para garantizar la continuidad de sus actuaciones.

- Seguimos sin tener una Ley General de Servicios Sociales en las que se establezcan unos mínimos para todos los ciudadanos, independientemente de su lugar de residencia y que establezca las bases del Sistema Público de Servicios Sociales, sin obstaculizar el respeto a la necesaria diferencia de cada territorio. La planificación de los Servicios Sociales presenta importantes diferencias según las distintas Comunidades Autónomas, si bien la práctica demuestra que esta planificación no responde, generalmente, tanto a un estudio previo de las necesidades sociales como a una distribución presupuestaria previa fundamentada en otro tipo de criterios. Quizá una de las posibles causas de este hecho sea la escasa participación de la población en los Servicios Sociales que sigue siendo mínima.

- Todas las Leyes de Servicios Sociales de las Comunidades Autónomas defienden el principio de universalidad pero realmente no hay un acuerdo generalizado al respecto existiendo diferentes argumentos en torno a esta cuestión e intensificándose este debate en este momento de crisis económica. Desde el punto de vista ideológico, hay quien defiende que la finalidad de la política social es la creación de una sociedad más justa y que la atención pública de las necesidades de todos los ciudadanos es la mejor manera de conseguirlo; otros, por el contrario, entienden que la política social debe cubrir las lagunas que se generan en el funcionamiento de la sociedad, asumiendo un papel residual a modo de red de seguridad. A nivel práctico, se 
defiende que los servicios universales son un derroche, al atender a personas que no necesitan el apoyo del Estado para cubrir sus necesidades, restando recursos a los más necesitados. En contra de este punto de vista creemos que es preferible ofertar servicios universales a los que contribuya económicamente el usuario en función de sus ingresos, ya que la existencia de servicios selectivos genera redes paralelas que no siempre cumplen los mismos estándares de atención, y que además provocan una mayor desigualdad y división social.

- Por otra parte, se ofrecen servicios «universales» sin adecuarse a las características específicas de determinados grupos de población, y como consecuencia de ello estas personas quedan excluidas de la posibilidad de beneficiarse de dichos servicios o actuaciones (por ejemplo: los cursos de formación del INEM en los que se exige un mínimo de formación básica de la que carecen muchos de los usuarios de los Servicios Sociales). Las acciones de discriminación positiva necesarias para facilitar el acceso a los servicios universales no son asimilables a las de carácter selectivo, ya que suponen el reconocimiento de derechos y la existencia de recursos adicionales para grupos en situación de especial necesidad o dificultad. La falta de este tipo de medidas de discriminación positiva obstaculiza la realización del principio de «normalización» y en este sentido encontramos ejemplos prácticos en las limitaciones a una integración real en el Sistema educativo ordinario de aquellas personas con dificultades de distinta índole. En otros casos, se han arbitrado las medidas necesarias, como ocurre con la supresión de barreras arquitectónicas o la reserva de puestos de trabajo para personas con discapacidades pero, en la práctica, no se controla suficientemente el cumplimiento de la normativa.

- La participación de la población en los Servicios Sociales se encuentra regulada en las Leyes de Servicios Sociales de las distintas Comunidades Autónomas a través de los denominados canales institucionales o Consejos de Bienestar. En muchas ocasiones y en opinión de los sectores sociales implicados, estos Consejos han sido bastante inoperantes al no disponer de infraestructura administrativa y financiera que permita un funcionamiento adecuado y actuar como meros órganos consultivos sin capacidad real de decisión. En algunos casos se critica su excesivo control por parte de la Administración que suele contar con una importante representación en los mismos. Por otro lado, la participacion de la población en la gestión directa de los Servicios es inexistente. La relación con los servicios es de simple usuario/consumidor, colocándole en una situación de inferioridad en la capacidad para decidir en aspectos importantes que le afectan directamente.

- Todavía los Servicios Sociales son percibidos por parte de la población como el lugar al que acudir para percibir meras ayudas econó- 
micas. En general no se hace Trabajo Social comunitario y predomina la idea de «gestoría social», como mucho con funciones asistenciales y reparadoras. Esta idea, que es una herencia del pasado, se ha reforzado porque se han asignado a los Servicios Sociales funciones correspondientes a otros Organismos (por ejemplo: tramitación de las Pensiones No Contributivas, Tarjeta de Asistencia Sanitaria, etc.). Es frecuente que la atención a las necesidades y problemas sociales se centre fundamentalmente en sus efectos, sin desarrollar actuaciones anticipadas orientadas a evitar que éstos se manifiesten y prevenir su aparición.

- En cuanto a la distribución competencial en materia de Servicios Sociales entre las distintas administraciones públicas, se habla en nuestro país, de una nueva centralización y control de las actividades de los Servicios Sociales por parte de las Comunidades Autónomas. Si bien este hecho favorece la garantía del equilibrio entre las distintas áreas que integran la Comunidad, no faltan las críticas por la falta de flexibilidad que, en ocasiones, tienen los gobiernos locales para diseñar sus propias estrategias de actuación en un intento de adecuarlas a las características propias de su demarcación. Parece que se ha logrado una descentralización desde la Administración Central a favor de la Administración Autonómica pero no acaba de ser realidad la descentralización hasta el nivel local.

- Esta distribución competencial hace necesario que se establezcan mecanismos efectivos de coordinación para evitar problemas como por ejemplo, superposición de servicios, lagunas en la atención a determinadas necesidades e incluso una actuación parcial en determinadas situaciones. Sin embargo, la falta de actuaciones conjuntas por parte de los distintos servicios y organismos públicos implicados y la ausencia de sistemas válidos y efectivos de coordinación entre todos ellos, impide llevar a cabo actuaciones globales e integrales. El afán por la búsqueda de protagonismo político obstaculiza en muchas ocasiones la realización de actuaciones conjuntas más eficaces. Estos problemas de coordinación se observan asimismo en cuanto a las relaciones de la Administración Pública con las Entidades sociales privadas.

- Salvo en algunas Comunidades Autónomas, no existe un marco legislativo adecuado que defina y respalde el papel del voluntariado en el campo de los Servicios Sociales. Existen todavía reticencias y temores por parte de los profesionales que trabajan en los Servicios Sociales públicos al interpretar que el trabajo de los voluntarios es exclusivamente un parche a la crisis económica y por lo tanto «mano de obra barata». Parece que en este sentido tenemos mucho que aprender de otros países con una cultura de solidaridad de larga tradición, en los que no se duda de la importante contribución de los voluntarios en la solución de muchos problemas sociales y en el desarrollo social de su comunidad. 
De lo comentado en los párrafos anteriores se deduce la necesidad de evaluar las actuaciones que en materia de Servicios Sociales se han venido realizando y tomar las decisiones adecuadas que permitan resituarlos en la línea de los principios que sirvieron de inspiración, teniendo en cuenta que cualquier análisis sobre la situación de los Servicios Sociales, en tanto instrumentos de la política social, exige tomar en consideración el contexto socioeconómico y político en que ésta se desarrolla.

La situación económica de crisis generalizada que vivimos y las transformaciones sociales recientes, están teniendo amplias repercusiones en todos los sistemas de protección europeos, y obviamente también en los españoles. El «mapa» de las necesidades aparece con rasgos nuevos, y como consecuencia de ello, los sistemas de protección, en un intento de adaptarse a la realidad, han de acometer las transformaciones necesarias. Ello ha provocado un amplio debate en el que, por ahora, no se vislumbran conclusiones compartidas. Sin ánimo de entrar en un profundo análisis, nadie discute que la crisis económica y sus lógicas repercusiones en los canales de financiación, las transformaciones en el mercado de trabajo, la evolución demográfica y el envejecimiento de la población, la modificación de las estructuras familiares, etc., son algunos de los factores que imponen determinadas modificaciones en los mecanismos de protección social.

La situación de crisis económica que estamos viviendo ha provocado la pérdida de gran número de empleos: el pasado mes de agosto, según los datos de Eurostat, España tenía un índice de desempleo del $21,2 \%$ de la población activa, cuando en ese mismo mes el índice de la Comunidad Europea era de un 10,4 \%. El descenso del empleo y el aumento del número de parados, unido a otros factores de gestión de la Seguridad Social, ha ocasionado un endurecimiento progresivo del acceso a las prestaciones sociales por desempleo. Otras áreas relacionadas con el bienestar como salud, vivienda y educación, también han reducido la posibilidad de acceso a su sistema de protección social debido a los recortes presupuestarios para contener el déficit público. Esto ha provocado un descontento generalizado en relación a la actual política social que ha sido contestada por todos los sectores sociales.

Como consecuencia de todo ello, y dado el aumento de necesidades sociales, se han desviado las demandas de protección social hacia los Servicios Sociales, que se están viendo desbordados ante una avalancha de problemas de todo tipo (de educación, de vivienda, de salud, etc.). Sin embargo, este incremento de la demanda de Servicios Sociales no se ha visto acompañado de un incremento paralelo en los presupuestos.

Estos ejemplos citados justifican el amplio debate que se plantea hoy en torno a los Servicios Sociales y que aborda aspectos tales 
como: la financiación, el papel de los Servicios Sociales en la redistribución del bienestar, responsabilidades de las distintas administraciones públicas, privatización de los Servicios Sociales, Servicios Sociales universales o selectivos, conveniencia de medidas de acción positiva para determinados colectivos o áreas geográficas, etc. La respuesta a todos estos aspectos afecta seriamente a los Servicios Sociales que, si bien han vivido en los últimos años un importante crecimiento desde el punto de vista cuantitativo, continúan sin tener el desarrollo alcanzado en cantidad y calidad en otros países que nos han servido de modelo. En definitiva, debemos seguir avanzando para llegar a consolidar unos Servicios Sociales que, organizados a nivel local, sean suficientes, flexibles y adaptados a cada circunstancia. 